\title{
Laboratory detection of the negative molecular ion $\mathrm{CCH}^{-}$
}

\author{
S. Brünken ${ }^{1}$, C. A. Gottlieb ${ }^{1}$, H. Gupta ${ }^{1,2}$, M. C. McCarthy ${ }^{1}$, and P. Thaddeus ${ }^{1}$ \\ 1 Harvard-Smithsonian Center for Astrophysics, 60 Garden Street, Cambridge, MA 02138, USA, and Division of Engineering and \\ Applied Sciences, Harvard University, 29 Oxford Street, Cambridge, MA 02138, USA \\ e-mail: sbruenken@cfa.harvard.edu \\ 2 Institute for Theoretical Chemistry, Department of Chemistry and Biochemistry, The University of Texas, Austin, TX 78712, USA
}

Received 19 December 2006 / Accepted 19 January 2007

\section{ABSTRACT}

\begin{abstract}
Aims. This paper reports the laboratory detection of the rotational spectrum of the acetylide anion, $\mathrm{CCH}^{-}$.
Methods. Five successive rotational transitions have been measured to high accuracy in a dc discharge with a free space millimeterwave spectrometer in the frequency range $83-417 \mathrm{GHz}$.

Results. Precise spectroscopic constants have been obtained for $\mathrm{CCH}^{-}$and transition rest frequencies can now be calculated to better than $1 \mathrm{~km} \mathrm{~s}^{-1}$ in equivalent radial velocity far into the terahertz region, allowing for deep astronomical searches for this anion in space. The highly stable and fairly polar $\mathrm{CCH}^{-}$anion is a likely candidate for radio astronomical detection, its neutral analogue $\mathrm{CCH}$ being among one of the most abundant molecules in a wide variety of astrophysical sources.
\end{abstract}

Key words. line: identification - molecular data - astrochemistry - methods: laboratory - ISM: molecules - radio lines: ISM

The search for negative molecular ions (anions) in astronomical sources has recently advanced in an unexpected way, driven by new laboratory results. In the radio band, we recently obtained the laboratory spectra of three carbon chain anions, $\mathrm{C}_{6} \mathrm{H}^{-}, \mathrm{C}_{4} \mathrm{H}^{-}$, and $\mathrm{C}_{8} \mathrm{H}^{-}$, and showed that the first is the carrier of the unidentified series of astronomical lines discovered by Kawaguchi et al. (1995) in the molecular envelope of IRC+10216 (McCarthy et al. 2006; Gupta et al. 2007). With good rest frequencies in hand, astronomical searches for $\mathrm{C}_{4} \mathrm{H}^{-}$and $\mathrm{C}_{8} \mathrm{H}^{-}$are now underway. The purpose of the present Letter is to describe the laboratory detection of a fourth molecular anion, $\mathrm{CCH}^{-}$, the shortest in the sequence of carbon chain anions with a closed-shell electronic ground state, and to give the rest frequencies required for a radio astronomical search for this polar, astronomically plausible molecule.

Neutral $\mathrm{CCH}$ was one of the first reactive molecules identified in space before its radio spectrum was observed in the terrestrial laboratory (Tucker et al. 1974). Since its initial detection in several star-forming regions and a circumstellar shell, its rotational spectrum has been studied in detail in the laboratory at millimeter and submillimeter wavelengths (Sastry et al. 1981; Gottlieb et al. 1983; Müller et al. 2000).

The $\mathrm{CCH}$ radical has been found to be one of the most abundant interstellar molecules in standard galactic sources. For example, in TMC-1, Orion A, and Sgr B2, its abundance is 10-15 times higher than that of the three-membered ring $c-\mathrm{C}_{3} \mathrm{H}_{2}$ and is only 2-3 times lower than that of formaldehyde $\left(\mathrm{H}_{2} \mathrm{CO}\right)$. In addition to the standard molecular sources, $\mathrm{CCH}$ is also observed in many other types of galactic sources including cold dust clouds (Wootten et al. 1980), planetary nebula (NGC 7027; Hasegawa \& Kwok 2001), photon-dominated regions (PDRs; Teyssier et al. 2004), diffuse clouds (Lucas \& Liszt 2000), and protoplanetary disks (Dutrey et al. 1997). In most astronomical sources the abundance of $\mathrm{CCH}$ is greater than that of $c-\mathrm{C}_{3} \mathrm{H}_{2}$, thereby confirming that, like $c-\mathrm{C}_{3} \mathrm{H}_{2}$, the $\mathrm{CCH}$ radical is also ubiquitous in our galaxy (Lucas \& Liszt 2000).

Because the lowest frequency transition of $\mathrm{CCH}^{-}$is at $83 \mathrm{GHz}$, well above the range of our very sensitive Fourier transform microwave spectrometer, we have only been able to study this anion with our less sensitive free space millimeterwave spectrometer (Gottlieb et al. 2003). Five successive rotational transitions have been detected in a dc discharge $(150 \mathrm{~mA})$ through a flowing mixture of acetylene $(85 \%)$ and argon $(15 \%)$ at a pressure of $<15$ mtorr when the discharge cell was cooled to $120 \mathrm{~K}$, the same conditions under which $\mathrm{C}_{4} \mathrm{H}^{-}$and $\mathrm{C}_{6} \mathrm{H}^{-}$ were observed (see Fig. 1). Lines of $\mathrm{CCH}^{-}$were about a factor of 25 less intense than the strongest hyperfine component of the corresponding rotational transition of $\mathrm{CCH}$ observed under the same conditions. Nevertheless, lines of $\mathrm{CCH}^{-}$were observed with a fairly high signal-to-noise ratio (Fig. 2), allowing frequencies to be measured to an accuracy of about $20 \mathrm{kHz}$ (Table 1). In the laboratory, the abundance of the anion is about $0.1 \%$ that of the neutral radical. For spectroscopic confirmation, three lines in both singly substituted ${ }^{13} \mathrm{C}$ species were observed when an isotopically enriched $\left(50 \% \mathrm{H}^{13} \mathrm{CCH}\right)$ sample was substituted for normal acetylene.

In much the same way as the anions in our previous papers were identified (McCarthy et al. 2006; Gupta et al. 2007), the lines in Table 1 can be assigned to $\mathrm{CCH}^{-}$with great confidence, because (i) they are closely harmonic in frequency, without evidence of hyperfine structure, as expected for the closed-shell configuration of $\mathrm{CCH}^{-}$(Ervin \& Lineberger 1991; Mladenovic et al. 1998); (ii) the rotational constant derived from the data is very close (within $0.01 \%$ ) to the published ab initio value for $\mathrm{CCH}^{-}\left(B_{0}=41636(20) \mathrm{MHz}\right.$; Mladenovic et al. 1998) and to that derived from our calculations (see Table 2); and (iii) the elemental composition of the discharge in which the lines are observed indicates that the carrier is a hydrocarbon or a pure carbon molecule, but the latter is ruled out by the disappearance 


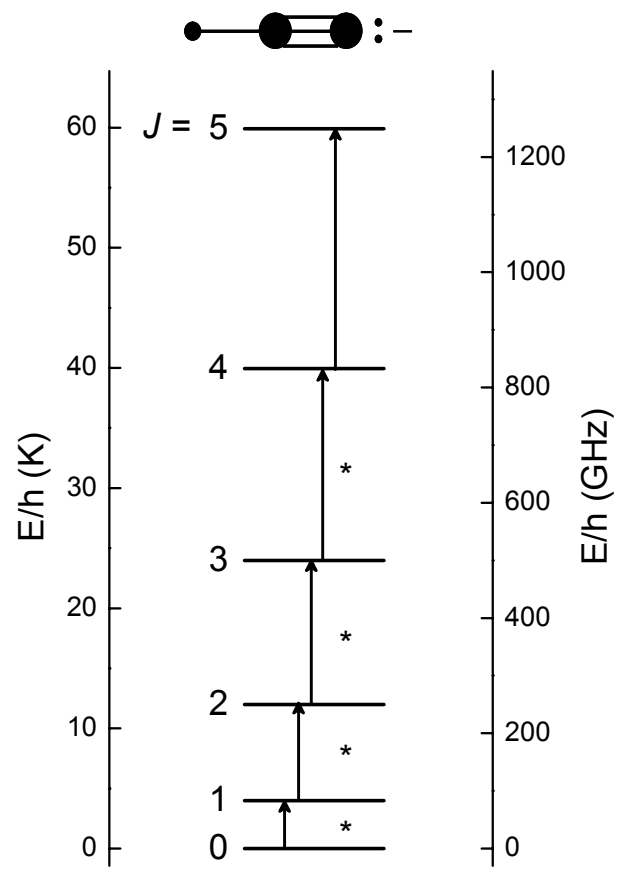

Fig. 1. Lower rotational energy levels of $\mathrm{CCH}^{-}$. The measured rotational transitions are indicated by arrows. Transitions that have been covered by published spectral line surveys towards various astronomical sources are marked with an asterisk.

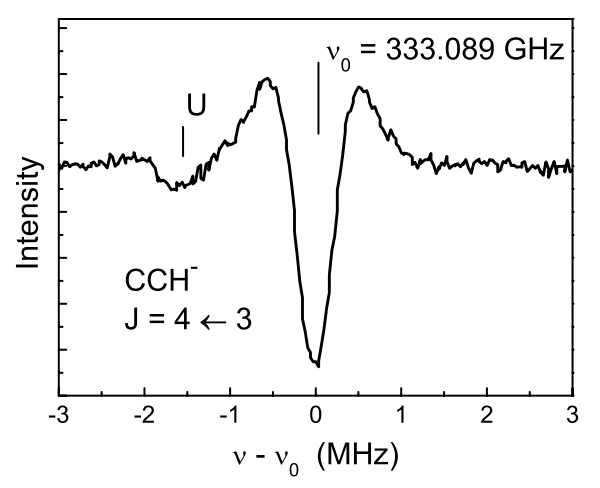

Fig. 2. The $J=4 \leftarrow 3$ rotational transition of $\mathrm{CCH}^{-}$observed with the free space millimeter-wave spectrometer in an observation of $10 \mathrm{~min}$. Owing to the modulation employed, the instrumental line shape is approximately the second derivative of a Lorentzian. The weak feature marked $\mathrm{U}$, which is produced by the discharge along with $\mathrm{CCH}^{-}$, is unidentified.

of the lines when D replaces $\mathrm{H}$ in the discharge; and finally, (iv) conclusive quantitative confirmation of the assignment is provided by detection of the two single-carbon-13 isotopic species at exactly the expected isotope shifts and with the predicted centrifugal distortion (see McCarthy et al. 1995).

The spectroscopic constants of $\mathrm{CCH}^{-}$(Table 2) allow for accurate calculation of transition frequencies far into the terahertz region. The sixth-order centrifugal distortion constant $H$ was not determined in the measurements here, but kept fixed at the theoretical value of Botschwina \& Sebald (2006); its contribution to the calculated line frequencies is less than $1 \mathrm{~km}^{-1}$ in equivalent
Table 1. Rotational frequencies of $\mathrm{CCH}^{-}$.

\begin{tabular}{crr}
\hline \hline$J^{\prime}-J^{\prime \prime}$ & $\begin{array}{r}\text { Frequency } \\
(\mathrm{MHz})\end{array}$ & $\begin{array}{r}\mathrm{O}-\mathrm{C}^{a} \\
(\mathrm{kHz})\end{array}$ \\
\hline $1-0$ & 83278.094 & 9 \\
$2-1$ & 166553.865 & 22 \\
$3-2$ & 249824.940 & -7 \\
$4-3$ & 333089.049 & -21 \\
$5-4$ & 416343.896 & 11 \\
\hline
\end{tabular}

Note. Estimated $1 \sigma$ uncertainties in the measured line frequencies are $20 \mathrm{kHz} .{ }^{a}$ Calculated from the spectroscopic constants in Table 2.

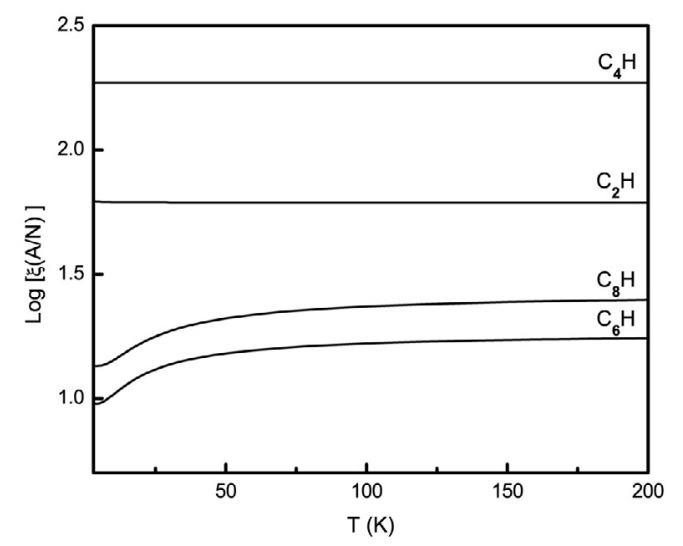

Fig. 3. Enhancement $(\xi)$ in the line intensity of the anion (A) relative to the corresponding neutral radical $(\mathrm{N})$. Plotted is the ratio of the square of the dipole moments divided by the partition functions. The ratio is independent of temperature for $\mathrm{CCH}$ and $\mathrm{C}_{4} \mathrm{H}$ owing to the ${ }^{2} \Sigma$ ground state of the neutral species, but depends on temperature for $\mathrm{C}_{6} \mathrm{H}$ and $\mathrm{C}_{8} \mathrm{H}$ in the ${ }^{2} \Pi$ ground state.

radial velocity for frequencies below $2 \mathrm{THz}$, comparable to the calculated uncertainty of the predictions. The present work now allows for deep searches for $\mathrm{CCH}^{-}$in essentially all astronomical sources.

As for the longer carbon chain anions, line intensities of the closed-shell $\mathrm{CCH}^{-}$benefit from the collapse of fine and hyperfine structure, and from the nearly four-fold larger dipole moment than that of the $\mathrm{CCH}$ radical (3.09 D for the anion (Mladenovic et al. 1998); versus 0.77 D for the neutral (Woon 1995)). As a result, lines of $\mathrm{CCH}^{-}$are roughly 50 times stronger than those of $\mathrm{CCH}$ per unit abundance, an effect that is largely independent of the rotational temperature of the source, as shown in Fig. 3, and facilitates the detection of the anion in space.

The $\mathrm{CCH}^{-}$anion seems to be a particularly good candidate for astronomical detection because of its high stability against photo detachment, owing to the high electron affinity of $\sim 3 \mathrm{eV}$ of neutral CCH (Rienstra-Kiracofe et al. 2002), but also because it fails to react with molecular hydrogen, the most abundant molecule in space. Gas phase reaction rate measurements by Barckholtz et al. (2001) yield upper limits of only $k<10^{-13} \mathrm{~cm}^{3} \mathrm{~s}^{-1}$ for the reaction of $\mathrm{CCH}^{-}$(and longer carbon chain anions) with $\mathrm{H}_{2}$, making this reaction a negligible destruction process for $\mathrm{CCH}^{-}$.

Recently Morisawa et al. (2005) performed a dedicated search for $\mathrm{CCH}^{-}$towards the cold dark cloud L134N and the star-forming region Sgr B2 (M) with the Nobeyama $45 \mathrm{~m}$ and the IRAM 30 m telescopes. Although their search was centered on a frequency calculated from the theoretical structure (Mladenovic et al. 1998), the $J=1-0$ line (83278.1 MHz, Table 1) lies 
Table 2. Spectroscopic constants (in $\mathrm{MHz}$ ) and dipole moment (in Debye) of $\mathrm{CCH}^{-}$.

\begin{tabular}{|c|c|c|c|c|c|c|c|}
\hline \multirow{3}{*}{ Constant } & \multicolumn{3}{|c|}{$\mathrm{CCH}^{-}$} & \multicolumn{2}{|c|}{${ }^{13} \mathrm{CCH}^{-}$} & \multicolumn{2}{|c|}{$\mathrm{C}^{13} \mathrm{CH}^{-}$} \\
\hline & \multirow[t]{2}{*}{ Laboratory } & \multicolumn{2}{|c|}{ Theoretical } & \multirow[t]{2}{*}{ Laboratory } & \multirow[t]{2}{*}{ Theoretical $^{a}$} & \multirow[t]{2}{*}{ Laboratory } & \multirow[t]{2}{*}{ Theoretical $^{a}$} \\
\hline & & This work $^{a}$ & Previous work $^{b}$ & & & & \\
\hline $\bar{B}$ & $41639.237(4)$ & 41636.1 & 41636 & $40111.413(7)$ & 40108.4 & $40637.441(5)$ & 40635.1 \\
\hline $10^{3} \mathrm{D}$ & 96.97(9) & 93 & 96.3 & $90.0(2)$ & 87 & $92.6(2)$ & 89 \\
\hline $10^{6} \mathrm{H}$ & {$[0.13]^{c}$} & - & 0.13 & & & & \\
\hline$\mu$ & & -3.1 & -3.1 & & & & \\
\hline
\end{tabular}

${ }^{a}$ From a quantum calculation (at the CCSD(T)/cc-pCVQZ level), corrected for vibration-rotation effects.

${ }^{b}$ From variational calculations (with the CCSD(T)/250 cGTO potential energy surface) (Mladenovic et al. 1998), $H$ was calculated with the same PEF by Botschwina \& Sebald (2006).

${ }^{c}$ Constrained to the theoretical value.

Table 3. Column density limits on $\mathrm{CCH}^{-}$.

\begin{tabular}{lrrrrrrrll}
\hline \hline Source & $\begin{array}{c}\text { Frequency } \\
(\mathrm{MHz})\end{array}$ & $\begin{array}{c}E_{\mathrm{up}} / k \\
(\mathrm{~K})\end{array}$ & $\begin{array}{c}\mu^{2} S \\
\left(\mathrm{D}^{2}\right)\end{array}$ & $\begin{array}{c}T_{r}^{*} \\
(\mathrm{mK})\end{array}$ & $\begin{array}{c}\Delta v \\
\left(\mathrm{kms}^{-1}\right)\end{array}$ & $\begin{array}{c}T_{\text {rot }} \\
(\mathrm{K})\end{array}$ & $\begin{array}{c}N_{\text {lim }} \\
\left(10^{12} \mathrm{~cm}^{-2}\right)\end{array}$ & Telescope & Ref. \\
\hline L134N & 83278 & 4 & 9.6 & 63 & 0.6 & 5 & 0.09 & Nobeyama 45 m & Morisawa et al. (2005) \\
Sgr B2 (M) & 83278 & 4 & 9.6 & 240 & 13 & 100 & 40 & Nobeyama 45 m & $\begin{array}{l}\text { Morisawa et al. (2005) } \\
\text { Nummelin et al. (1998) }\end{array}$ \\
& 249825 & 24 & 28.7 & 100 & 20 & 100 & 3.0 & SEST 15 m & $\begin{array}{l}\text { Numb } \\
\text { IRC +10216 }\end{array}$ \\
\hline 333089 & 40 & 38.3 & 200 & 20 & 100 & 4.0 & CSO 10.4 m & Sutton et al. (1991) \\
& 83278 & 4 & 9.6 & 5 & 30 & 20 & 0.4 & IRAM 30 m & Cernicharo (2006) $^{a}$ \\
& 166554 & 12 & 19.1 & 100 & 30 & 20 & 3.0 & IRAM 30 m & Cernicharo et al. (2000) \\
Orion KL & 333089 & 40 & 38.3 & 200 & 30 & 20 & 6.0 & CSO 10.4 m & Groesbeck et al. (1994) \\
& 83278 & 4 & 9.6 & 50 & 5 & 10 & 0.4 & NRAO 11 m & Turner (1991) \\
& 333089 & 40 & 38.3 & 500 & 5 & 10 & 9.5 & CSO 10.4 m & Schilke et al. (1997) \\
\hline
\end{tabular}

${ }^{a}$ Corresponds to published data by Cernicharo et al. (2004).

within their bandwidth. Our laboratory measurements show that the weak feature that Morisama et al. observed towards L134N is not $\mathrm{CCH}^{-}$: the frequency of this astronomical line differs from the measured frequency by $\sim 7 \mathrm{MHz}$, or 60 times the linewidth in this source. From the noise level at the $\mathrm{CCH}^{-}$line position we estimate that the anion/neutral ratio is $<0.2 \%$ in L134N on the assumption that $N(\mathrm{CCH})=5 \times 10^{13} \mathrm{~cm}^{-2}$ (Dickens et al. 2000). Similarly, the upper limit for this ratio in Sgr B2 (M) is $<5 \%$ for $N(\mathrm{CCH})=7.8 \times 10^{14} \mathrm{~cm}^{-2}$ (Sutton et al. 1991).

We found no evidence of $\mathrm{CCH}^{-}$in published spectral line surveys of standard molecular galactic sources. Limits on the column densities of $\mathrm{CCH}^{-}$are summarized in Table 3. In Sgr B2 (M), a better constraint on the anion-to-neutral ratio of $<0.4 \%$ is obtained from an upper limit for the 3-2 transition of $<100 \mathrm{mK}$ (Nummelin et al. 1998): $N\left(\mathrm{CCH}^{-}\right)<3.0 \times$ $10^{12} \mathrm{~cm}^{-2}$. Lines of $\mathrm{C}_{6} \mathrm{H}^{-}$are quite strong in $\mathrm{IRC}+10216$, so we also examined published surveys of this source (Cernicharo et al. 2000; Groesbeck et al. 1994; Cernicharo et al. 2004). The best limit for $\mathrm{CCH}^{-}$is the one obtained from the 1-0 transition (Cernicharo 2006; Cernicharo et al. 2004), which yields $N\left(\mathrm{CCH}^{-}\right)<4 \times 10^{11} \mathrm{~cm}^{-2}$. The ratio of the anion to the neutral in IRC +10216 of $<0.01 \%$ (for an assumed column density of $\mathrm{CCH}$ of $5 \times 10^{15} \mathrm{~cm}^{-2}$; Cernicharo et al. 2000), is more than two orders of magnitude smaller than that found for $\mathrm{C}_{6} \mathrm{H}^{-}$. Unfortunately there is no survey data above $50 \mathrm{GHz}$ in TMC-1, the other source in which $\mathrm{C}_{6} \mathrm{H}^{-}$has been found. In Orion $\mathrm{A}$, the $\mathrm{CCH}$ radical is quite abundant $\left(N(\mathrm{CCH})=(3-4) \times 10^{14} \mathrm{~cm}^{-2}\right.$; Ziurys et al. 1982), so the spectral line surveys were examined for possible evidence of $\mathrm{CCH}^{-}$. The 1-0 and 3-2 transitions of $\mathrm{CCH}^{-}$were covered in two separate surveys of Orion KL (Turner 1991; Schilke et al. 1997). On the assumption that $\mathrm{CCH}^{-}$ is present in the same extended region as $\mathrm{CCH}$, we estimate that $N\left(\mathrm{CCH}^{-}\right)<4 \times 10^{11} \mathrm{~cm}^{-2}$ and the anion/neutral ratio is $<0.1 \%$. From the published spectra in L134N, Orion KL, and Sgr B2 (M), our limit for the ratio of $\mathrm{CCH}^{-}$to $\mathrm{CCH}$ is $<(0.1-0.4) \%$, which is about 10 times higher than that derived for IRC+10216.

In conclusion, we find anion-to-neutral ratios for $\mathrm{CCH}^{-}$in all investigated sources of more than one order of magnitude lower than found for the longer chain $\mathrm{C}_{6} \mathrm{H}^{-}$, which is about a few percent in IRC+10216 and TMC-1. This result is consistent with a simple set of assumptions about the formation of these two anions, specifically: (i) both $\mathrm{CCH}^{-}$and $\mathrm{C}_{6} \mathrm{H}^{-}$result from the competition between electron attachment to the parent neutral molecule and subsequent photodetachment; (ii) the photodetachment rates for $\mathrm{CCH}^{-}$and $\mathrm{C}_{6} \mathrm{H}^{-}$do not much differ, because the electron affinities for $\mathrm{CCH}(2.969 \mathrm{eV})$ and $\mathrm{C}_{6} \mathrm{H}(3.809 \mathrm{eV})$ are not very different (Rienstra-Kiracofe et al. 2002); and (iii) the electron attachment rate for $\mathrm{CCH}$, however, is much lower than that for $\mathrm{C}_{6} \mathrm{H}$, because of the much lower density of excited vibrational states. To clarify this simple picture, or to rule it out, much better astronomical observations than those now available are needed. Radio surveys of a variety of molecular sources at the frequencies given here are an obvious place to start. Deep integrations should improve the present limits by half an order of magnitude or more, or may ultimately yield the detection of $\mathrm{CCH}^{-}$in space.

Acknowledgements. We gratefully acknowledge continuing conversations with A. Dalgarno, E. Herbst, and W. Klemperer on the subject of negative ions in space and related issues. We thank J. F. Stanton for guidance with the quantum calculations and K. Higgins for the use of his workstations. We are grateful to J. Cernicharo and P. Botschwina for providing us with unpublished data. This work is supported by National Science Foundation Grant CHE-0353693; additional support is provided by the Robert A. Welch Foundation through a grant to J. F. Stanton at the University of Texas. S. B. is grateful to the Harvard College Observatory for the 2005 Menzel fellowship. 


\section{References}

Barckholtz, C., Snow, T. P., \& Bierbaum, V. M. 2001, ApJ, 547, L171

Botschwina, P., \& Sebald, P. 2006, private communication

Cernicharo, J. 2006, private communication

Cernicharo, J., Guélin, M., \& Kahane, C. 2000, A\&AS, 142, 181

Cernicharo, J., Guélin, M., \& Pardo, J. R. 2004, ApJ, 615, L145

Dickens, J. E., Irvine, W. M., Snell, R. L., et al. 2000, ApJ, 542, 870

Dutrey, A., Guilloteau, S., \& Guelin, M. 1997, A\&A, 317, L55

Ervin, K. M., \& Lineberger, W. C. 1991, J. Phys. Chem., 95, 1167

Gottlieb, C. A., Gottlieb, E. W., \& Thaddeus, P. 1983, ApJ, 264, 740

Gottlieb, C. A., Myers, P. C., \& Thaddeus, P. 2003, ApJ, 588, 655

Groesbeck, T. D., Phillips, T. G., \& Blake, G. A. 1994, ApJS, 94, 147

Gupta, H., Brünken, S., Tamassia, F., et al. 2007, ApJ, 655, L57

Hasegawa, T. I., \& Kwok, S. 2001, ApJ, 562, 824

Kawaguchi, K., Kasai, Y., Ishikawa, S.-I., \& Kaifu, N. 1995, PASJ, 47, 853

Lucas, R., \& Liszt, H. S. 2000, A\&A, 358, 1069

McCarthy, M. C., Gottlieb, C. A., \& Thaddeus, P. 1995, J. Mol. Spectrosc., 173, 303

McCarthy, M. C., Gottlieb, C. A., Gupta, H., \& Thaddeus, P. 2006, ApJ, 652, L141
Mladenovic, M., Botschwina, P., Sebald, P., \& Carter, S. 1998, Theor. Chem. Acc., 100, 134

Morisawa, Y., Hoshina, H., Kato, Y., et al. 2005, PASJ, 57, 325

Müller, H. S. P., Klaus, T., \& Winnewisser, G. 2000, A\&A, 357, L65

Nummelin, A., Bergman, P., Hjalmarson, A., et al. 1998, ApJS, 117, 427

Rienstra-Kiracofe, J. C., Tschumper, G. S., Schaefer III, H. F., Nandi, S., \& Ellison, G. B. 2002, Chem. Rev., 102, 231

Sastry, K. V. L. N., Helminger, P., Charo, A., Herbst, E., \& De Lucia, F. C. 1981, ApJ, 251, L119

Schilke, P., Groesbeck, T. D., Blake, G. A., \& Phillips, T. G. 1997, ApJS, 108, 301

Sutton, E. C., Jaminet, P. A., Danchi, W. C., \& Blake, G. A. 1991, ApJS, 77, 255

Teyssier, D., Fossé, D., Gerin, M., et al. 2004, A\&A, 417, 135

Tucker, K. D., Kutner, M. L., \& Thaddeus, P. 1974, ApJ, 193, L115

Turner, B. E. 1991, ApJS, 76, 617

Woon, D. E. 1995, Chem, Phys. Lett., 244, 45

Wootten, A., Bozyan, E. P., Garrett, D. B., Loren, R. B., \& Snell, R. L. 1980, ApJ, 239, 844

Ziurys, L. M., Saykally, R. J., Plambeck, R. L., \& Erickson, N. R. 1982, ApJ, 254, 94 